\title{
Northern Region asplenia register-analysis of first two years
}

\author{
G P Spickett, J Bullimore, J Wallis, S Smith, P Saunders
}

\begin{abstract}
Objectives-To assess the feasibility of setting up a register of patients with asplenia within a defined geographical area; to ensure that guidelines on best practice were implemented; to obtain information on antibody levels to pneumococcal capsular polysaccharides and Haemophilus influenzae type b capsular polysaccharide, before and after immunisation and annually thereafter; to raise awareness of risks among clinicians and to offer advice on management.
\end{abstract}

Design-Prospective recruitment using multiple sources of recruitment. Annual follow up reminders sent from Registration Centre.

Subjects-Population of (old, pre-1995) Northern Health Region: approximately 3.1 million.

Main outcome measures-Data were obtained on reasons for asplenia, duration of asplenia, use of prophylactic antibiotics, Medic-Alert bracelets, immunisations, antibody levels, death.

Results-The register was initiated at the beginning of April 1995 and ran to the end of March 1997. After two years of operation, 1111 cases had been registered but the response from some health districts was poor. Major primary causes of asplenia were trauma (264), other surgical (198), lymphoproliferative disease (154), and idiopathic thrombocytopenic purpura (147). There were 664 patients on prophylactic antibiotics, of whom 498 were on continuous antibiotics. Only 18 had any type of warning bracelet. Antibody measurements were carried out at least once on $75 \%$ of patients; 306 patients had satisfactory antibody levels on first blood sample in year 1 , rising to 405 in year $2 ; 43$ patients failed to make any antibody response to Pneumovax despite multiple immunisations, and three patients failed to respond to Hib vaccine. Sixteen patients with satisfactory antibody levels in year 1 had low levels in year 2 requiring vaccine boosters. Sixteen deaths were reported, two of which were directly attributable to overwhelming sepsis.

Conclusions-Registration has been successful and has raised awareness of the management of asplenia. Compliance with antibiotic prophylaxis and immunisation was initially poor. A potential high risk group of vaccine non-responders has been identified and poor persistence of pneumococcal antibodies has been identi- fied which is likely to alter approaches to immunisation in asplenic patients. (f Clin Pathol 1999;52:424-429)

Keywords: asplenia; immunodeficiency; immunisation

Optimum management of asplenia has been widely debated over the last six years and current approaches to management have often not been supported by strong clinical evidence. Various authoritative reviews have suggested approaches to management ${ }^{1-4}$ and guidance has also been issue by the chief medical officer to all doctors in England. The main concern has been the possibility of death from overwhelming sepsis. However, the actual risk of this has never been ascertained over large long term series. A study in Australia on 1490 patients receiving no prophylaxis identified an incidence of serious sepsis of $0.42 / 100$ person-years. ${ }^{5}$ Most data have indicated that the risk of sepsis is greatest in the three years immediately after loss of the spleen ${ }^{6}$ and that the risk of infection is higher in children, ${ }^{7}$ but there is some evidence to suggest that the risk is lifelong. ${ }^{8}$ The evidence that interventions such as continuous antibiotic prophylaxis or immunisation affect the risk of overwhelming sepsis has never been seriously addressed in large controlled trials, and the shortcomings of current advice and practice have been openly challenged. ${ }^{9}$ The value of antibiotics has only been shown in children with sickle cell disease, and the value of immunisation never formally evaluated. Despite this uncertainty, the recently published British Society for Haematology (BSH) consensus guidelines of current best practice still recommend antibiotics and immunisation. ${ }^{10}$

Following on from the presentation in quick succession of three asplenic patients with overwhelming sepsis to hospitals in Newcastle, ${ }^{11}$ none of whom had received immunisations, antibiotic advice, or any counselling, a meeting of interested parties was held to produce local guidelines for the management of asplenic patients. Following an extended debate a set of agreed guidelines was produced (Appendix 1). These predated the publication of the $\mathrm{BSH}$ guidelines, ${ }^{10}$ although access was obtained to an early draft to ensure that local policy would not diverge radically from the national consensus. The regional haematologists' organisation was keen to see a register of such patients. Accordingly, it was agreed that the department of immunology would establish such a register and undertake serial monitoring of antibody levels in order to obtain information on the response to the vaccines in an asplenic population and on the persistence of responses in the long term. It was felt that a proactive register
Accepted for publication 8 February 1999 


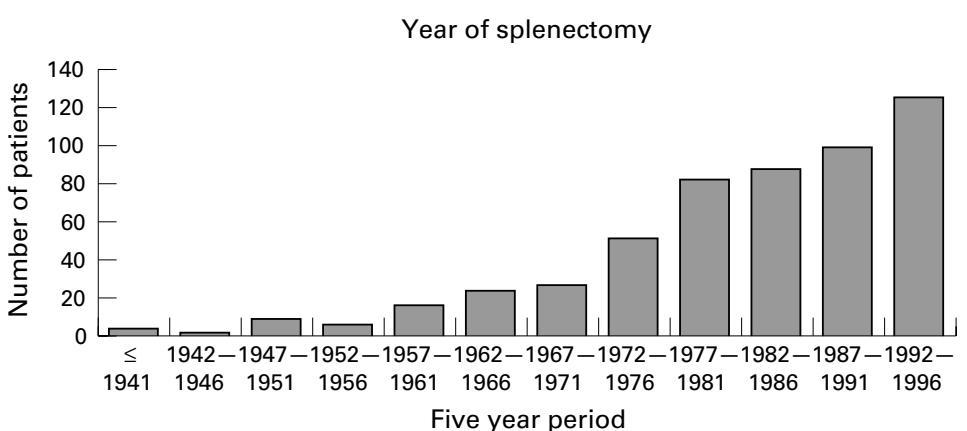

Figure 1 Year of splenectomy by five year periods. For the four patients splenectomised before 1941 the years were: 1927, 1930, 1940, and 1941.

would serve to raise awareness of the problem and would of itself improve management.

\section{Methods}

Information on the locally produced guidelines and the register, together with appropriate registration forms were sent to all hospitals in the region (chief executives and medical directors) and to all family health services authority (FHSA) medical advisers, with the request that they support the project by disseminating information to all general practices within their district. Information was also distributed to all consultant haematologists within the region.

Following receipt of registration forms, the registering doctor was sent a request for a blood sample on the patient to measure antibodies to pneumococcal polysaccharide and Haemophilus influenzae type $\mathrm{b}$ (Hib antibodies). A laminated warning card (Appendix 2) was also provided. This card is personalised for each patient, unlike the later Department of Health card.

Serum antibodies were measured routinely, initially in the regional immunology laboratory, Newcastle upon Tyne (pneumococcal antibodies) and the regional immunology laboratory, Churchill Hospital Oxford (Hib antibodies), but latterly all measurements have been carried out in Newcastle. Solid phase assays, calibrated against common standards, have been used throughout. Both laboratories participate in the European quality control scheme for specific antibodies. ${ }^{12}{ }^{13}$ All reports were scrutinised by one person (GPS) before dispatch, to ensure consistency of advice on immunisation.

Antibodies to pneumococcal polysaccharide were measured using 23-valent pneumococcal polysaccharide (Pneumovax-II), without adsorption of the cell wall polysaccharide. No agreed international standard exists for pneumococcal polysaccharide antibodies, although there is an unofficial standard in the United Kingdom.

Hib antibodies were measured initially using pure Hib capsular polysaccharide, obtained from Pasteur-Merieux, and the assay was calibrated against an agreed international standard, with assigned absolute unitage. More recently a commercial assay, provided by Baxter-Immuno, has been used after comparative studies against the original assay.

Arbitrary levels of antibodies were set, below which protection was deemed dubious and further immunisation advised. There are no studies which confirm satisfactorily the absolute protective level of pneumococcal polysaccharide antibodies, but it is known in children that the protective level of Hib antibodies is $1 \mu \mathrm{g} / \mathrm{ml}$ from multiple large vaccine trials. ${ }^{13}$ As a safety net for advice on reimmunisation and for the purposes of analysis of the results, arbitrary levels were set for each antibody. These were $35 \mathrm{U} / \mathrm{ml}$ for pneumococcal antibodies and $1.5 \mu \mathrm{g} / \mathrm{ml}$ for Hib antibodies. Where antibody levels were below these predefined levels, advice on immunisation was given and a further postimmunisation blood sample requested. On the annual anniversary of registration, a further blood sample was requested on all patients. Advice on reimmunisation was given according to levels.

Patients were not seen in a central clinic unless specifically and separately referred. Small numbers of patients were referred to general practitioners for advice and management, usually where there were problems of antibiotic or vaccine intolerance/allergy or where the patient was reluctant to consider any prophylaxis.

Any deaths reported to the registry were followed up to ascertain whether sepsis had played a major role in the death.

The data were analysed at the end of two years. However, because of the rolling nature of registration, only a proportion of patients have been through the full two year cycle.

\section{Results}

REGISTRATION

The data presented here relate to the period from initiation of the study in April 1995 until the end of March 1997. After 24 months, 1111 patients had been registered. These included 73 recently registered patients on whom little information was available at the time of analysis. Response to requests to report asplenic patients was variable between districts within the region, and was in part related to the organisation and interests of general practitioner groups and to whether haematologists were single handed. Figure 1 shows the duration of splenectomy before registration. Figure 2 shows the reason for asplenia. "Other surgical" in this context covers spleens removed either deliberately for other surgical conditions including gastric and pancreatic surgery or owing to intraoperative trauma to the spleen. "Other" covers a miscellany of medical conditions but includes patients with functional asplenia secondary to coeliac disease. Thirty two children were included on the register, 17 of whom had splenectomies for hereditary spherocytosis. Not all registration forms submitted contained complete data, and the usual information missing was immunisation history. No attempt was made to obtain original clinical records, nor to confirm pathological diagnoses at splenectomy.

No attempt was made to collect follow up data on the patients to assess whether there had been a change in practice with respect to immunisation, antibiotics, or carriage of warning cards, as it was felt that this would overburden general practitioners who were responsible for the majority of registrations. Equally, no attempt 
Reason for asplenia

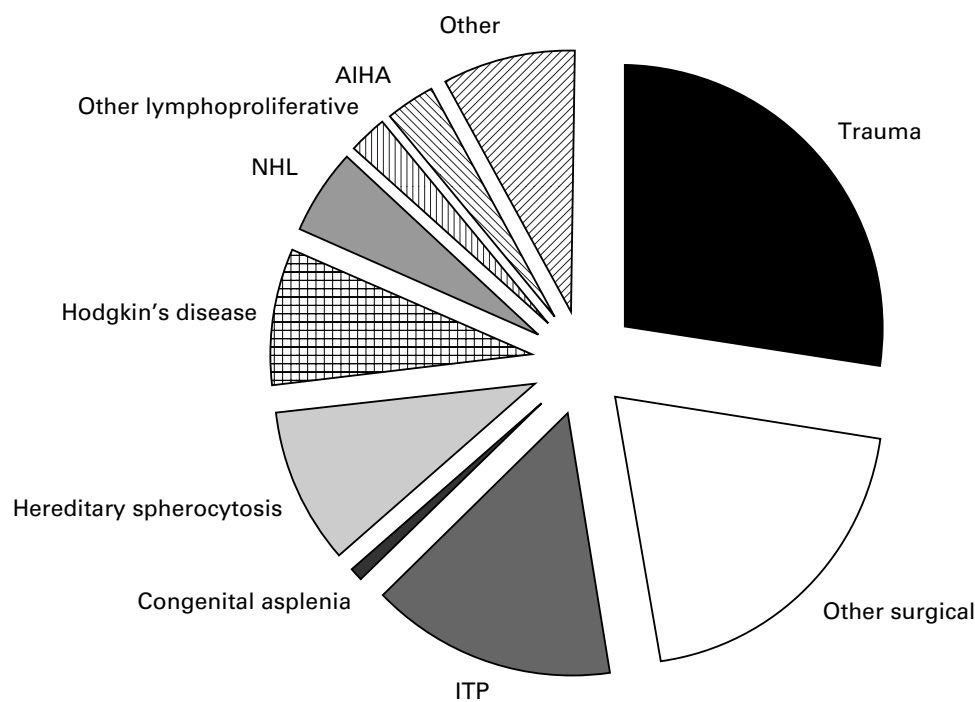

Figure 2 Breakdown of the reason for asplenia. "Other surgical" covers mainly splenectomy as part of gastric or pancreatic surgery, or through surgical trauma. ITP, idiopathic thrombocytopenic purpura; NHL, non-Hodgkin lymphoma; AIHA, autoimmune haemolytic anaemia. "Other lymphoproliferative" includes 14 patients with hairy cell leukaemia, six with chronic lymphocytic leukaemia, and three others. "Other" includes patients with myeloproliferative disease, spontaneous rupture, coeliac disease, splenic cysts or abscesses, metabolic diseases, vascular abnormalities, and other congenital diseases (sickle cell anaemia, thalassaemia, etc).

was made to follow up those patients where follow up blood samples were not received.

\section{ANTIBIOTIC PROPHYLAXIS}

Antibiotic prophylaxis was being taken by 664 patients at the time of registration, while 257 were on no regular prophylaxis. Of the 664 patients receiving antibiotic prophylaxis, 498 were reported to be on continuous antibiotics, while 36 were on intermittent antibiotics; a further 137 were reported to have a standby supply at home; some of these patients had both intermittent antibiotics and a standby supply. Antibiotics prescribed were penicillin V (546), 71 amoxycillin (amoxicillin) (71), and erythromycin (52). Only 18 patients had any kind of warning bracelet (Medic-Alert or equivalent) at the time of first registration.

IMMUNISATION

Three hundred and nine patients $(28 \%)$ were given Pneumovax before splenectomy, 115 were given $\mathrm{Hib}$ vaccine $(10 \%)$, and 61 meningococcal $\mathrm{A}$ and $\mathrm{C}$ vaccine $(5 \%)$. At least one blood sample was received on $75 \%$ of all patients registered. Of the patients first registered in year 1, 306 had adequate antibody levels to both pneumococcal and haemophilus polysaccharides; when patients registered in year 2 are included the figure rises to 405 . In this context "adequate" means pneumococcal antibodies $\geqslant 35 \mathrm{U} / \mathrm{ml}$ and for $\mathrm{Hib}$ antibodies $\geqslant 1.5 \mu \mathrm{g} / \mathrm{ml}$.

ANTIBODY LEVELS

Table 1 shows the distribution of antibody levels to pneumococcal polysaccharide on the first blood sample, while table 2 shows the equivalent data for Hib. One hundred and fifty three patients $(14 \%)$ of patients had inadequate antibodies to pneumococcal polysaccharide while 275 (25\%) had inadequate Hib antibodies. This latter figure probably reflects the lower awareness of the possible value of $\mathrm{Hib}$ immunisation in this patient group.

Forty three patients (4\%) had blood levels indicating a failure to respond to Pneumovax. Table 3 gives the diagnostic breakdown of these non-responders. Three patients failed to respond to Hib immunisation-one with hairy cell leukaemia, one with autoimmune haemolytic anaemia, and one with chronic myeloid leukaemia. In this context, non-responders have no rise in antibody titre after immunisation compared with before immunisation (or a less than twofold increase). Advice was given to reporting doctors on the need for further immunological investigations and the likely risk of infection, but no further investigations of immune function were done within the context of the study. Sixteen patients whose pneumococcal antibodies were satisfactory in year 1 had levels that had fallen to $\leqslant 35 \mathrm{U} / \mathrm{ml}$ in year 2 and therefore required immunisation. In 102 patients with low antibodies to one or both antigens, immunisation and rebleeding was advised, but no follow up blood samples were received, despite repeated requests. There were no differences in immunisation status or antibody levels between patients reported to the register by general practitioners, compared with those reported by hospital doctors.

No adverse effects from vaccination were reported to the register.

DEATHS

Sixteen deaths were notified. Two were directly caused by overwhelming sepsis. The first case had been splenectomised in 1989 for idiopathic thrombocytopenic purpura and given

Table 1 Distribution of antipneumococcal antibodies

\begin{tabular}{ll}
\hline Pneumococcal antibody level (U/ml) & Number of patients \\
\hline$\leqslant 20$ (no protection) & 84 \\
$>20$ but $<35$ (suboptimal protection) & 69 \\
$\geqslant 35$ but $<100$ & 220 \\
$\geqslant 100$ (high) & 264 \\
\hline
\end{tabular}

Table 2 Distribution of anti-Haemophilus influenzae type $b$ (Hib) antibodies

\begin{tabular}{ll}
\hline Hib antibody level $(\mu \mathrm{g} / \mathrm{ml})$ & Number of patients \\
\hline$\leqslant 1.0$ (no protection) & 210 \\
$>1.0$ but $<1.5$ (suboptimal protection) & 65 \\
$\geqslant 1.5$ but $<10$ & 276 \\
$\geqslant 10$ (high) & 89 \\
\hline
\end{tabular}

Table 3 Diagnoses of Pneumovax non-responders

\begin{tabular}{ll}
\hline Diagnosis & Number of patients \\
\hline Trauma & 9 \\
Hodgkin disease & 5 \\
Thrombocytopenic purpura & 5 \\
Other surgical & 4 \\
No diagnosis given & 4 \\
Non-Hodgkin lymphoma & 3 \\
Chronic lymphocytic leukaemia & 2 \\
Splenic abscesses & 2 \\
Autoimmune haemolytic anaemia & 2 \\
Other & 7 \\
\hline
\end{tabular}


Pneumovax in 1992. He was not on regular antibiotics and died from pneumococcal meningitis. Blood samples taken shortly before death showed very low anti-pneumococcal antibodies. The second case was splenectomised in 1997 for autoimmune haemolytic anaemia and died one month later from septicaemia despite antibiotics and immunisation. No blood samples were received. Eight patients died from underlying malignant disease, one died from surgical complications of splenectomy, one with a splenectomy for trauma died from the trauma, one patient died from a stroke, and in three patients no cause of death was ascertained.

\section{Discussion}

The analysis of the first two years' data from this project has shown that the establishment of a proactive registration scheme for asplenic patients is feasible and that it has on the whole been well received by participating doctors.

In 1995 MacInnes et al reported their experience in South Buckinghamshire of setting up a local register. ${ }^{14}$ This report did not include the measurement of antibody levels but concentrated on ensuring immunisation and antibiotic prophylaxis. At the time of first contact these investigators found that only $9 \%$ of adults were taking antibiotic prophylaxis. Subsequently this group has also been measuring antibody levels. At the time of first registration on our register, significant numbers of patients $(23 \%)$ were not receiving antibiotic prophylaxis either continuously or intermittently, and had not been vaccinated. Vaccination rates against $\mathrm{Hib}$ and meningococcus were lower than for pneumococcus, owing to lower awareness of the possible value of these vaccinations in this group. It is intended to reaudit the provision of prophylactic antibiotics specifically to assess the impact of the register. As there are likely to be compliance problems with long term treatment, this is an area that will require regular review through the register. Negligible numbers of patients had any form of personal warning material and this was rectified by the provision through the register of a laminated warning card personalised for each patient.

"Protective" levels of antibodies have been well defined only for Hib, where a level of 1.0 $\mu \mathrm{g} / \mathrm{ml}$ has been shown to be protective in large vaccine trials. ${ }^{13}$ For pneumococcal antibodies, the situation is more complicated in that Pneumovax contains the polysaccharides of 23 different serotypes which vary in their immunogenicity, and also contains cell wall polysaccharide which is immunogenic but is not thought to confer protection against invasive disease. A good response to one serotype may therefore mask a failure of responsiveness to other serotypes, as only total "antiPneumovax" antibodies are measured (it is impracticable to measure serotype specific antibodies on such a large cohort). A level of 20 $\mathrm{U} / \mathrm{ml}$ is considered to be the cut off for total pneumococcal antibodies. However, to ensure that adequate protection is present an arbitrary cut off greater than the minima was set for both antibodies: immunisation was advised if the pneumococcal antibody level was $\leqslant 35 \mathrm{U} / \mathrm{ml}$ or the Hib antibody $\leqslant 1.5 \mu \mathrm{g} / \mathrm{ml}$. Blanket immunisation of all patients was not recommended, lest those with high levels of antibodies-either from natural exposure or previous undocumented immunisation-be immunised and have adverse reactions.

Konradsen et al have carried out a similar but smaller study on immunisation responses of Danish asplenic patients. ${ }^{15}$ The results are not directly comparable for two reasons: first, the lack of international standards for pneumococcal antibodies; and second, differences in the target antigens used in the solid phase assays for pneumococcal and Hib antibodies. They also set different target ranges for "protective antibodies." With these points in mind, they identified that $37 \%$ of their cohort had "protective" antibodies to six pneumococcal serotypes and Hib. They achieved only $4 \%$ follow up blood samples on their patients.

Seventy five per cent of patients have had at least one measurement of antibodies carried out. Patients with low antibody levels have been advised to receive further immunisations, and blood levels after immunisation have identified small numbers of patients who have failed to respond, often to multiple doses. These patients may constitute a high risk group for sepsis, although long term follow up of such patients will be required to prove this. Although this group included some patients with lymphoproliferative disease, as might be expected, there were also patients with other diagnoses where failure of immunisation response would not necessarily be expected. There is some evidence that some patients without spleens have impaired immune responsiveness, particularly to polysaccharide antigens. ${ }^{16}{ }^{17}$ Sullivan et al looked at 31 patients with splenectomy or functional asplenia and found normal secondary responses to the older Pneumovax antigen in all patients except those with Hodgkin disease. ${ }^{18}$ Primary (IgM) responses to the bacteriophage $\phi \mathrm{X} 174-\mathrm{a}$ neoantigen that allows the primary and secondary immune response to be studiedwere impaired in all patients, and in the secondary response there was a poor switch from $\operatorname{IgM}$ to $\operatorname{IgG}$ production. The lack of responsiveness in some patients, who are not necessarily predictable, is of concern lest a false sense of security occurs following immunisation without checking postimmunisation levels. Further, pneumococcal sepsis may occur with strains not represented in the vaccine: this may account for up to $15 \%$ of cases of pneumococcal sepsis. ${ }^{19}$

The other major question that requires answering is how often immunisations should be given. The original work in immunocompetent patients has suggested that antibodies to pneumococcal polysaccharides remain increased for at least 10 years, ${ }^{20}$ although it appears that splenectomised patients may lose their antibody faster and require reimmunisation after three to five years. ${ }^{21}$ No large scale data have addressed this problem, and the cohort that we have assembled should allow us 
to obtain a better picture, provided that support for the register is maintained. This in turn will allow assessment of the role of serum antibody measurement, the value of which has been questioned. ${ }^{9}$ The preliminary data suggest that some patients do not maintain high pneumococcal antibody levels following immunisation, while Hib antibodies are more likely to remain raised.

There has been good uptake of the antibody monitoring service, which has been provided at no charge to the registering doctor for the first three years. However, no further regional/ supradistrict funding has been allocated to the project despite application, meaning that fees will have to be charged to maintain the register. This is likely to reduce its effectiveness as a monitoring tool and discourage doctors from registering patients. Activities of this type operate more effectively with central funding, allowing the service to be free at the point of use by clinicians. As most samples are sent in by general practitioners who do not normally deal with the (distant) laboratory, this increases the workload of individual billing and dramatically increases the overheads for the service. With the change in contracting to general practitioner led discussions, this may become easier, although arrangements would have to be made with a large number of individual primary care groups. Central funding would also enable local efforts such as those in South Buckinghamshire and the Northern Region to be coordinated and extended.

Clearly an opportunity to provide crucial long term data on therapeutic interventions which are widely used but have never been formally proven will be lost if registers are not continued. The follow up period so far on our study is too short to show whether the currently recommended immunisation and prophylactic antibiotic schedule is of value in preventing sepsis, but it does indicate that this sort of approach is feasible at a regional level. Only two deaths related to infection have occurred, one in a patient immunised but not on antibiotics and one both immunised and on antibiotics. In the later case, infection occurred early, which has always been considered a risk period.

We are grateful to the (late!) Northern Regional Health Authority for funding this project for the first two years. We also wish to thank all the general practitioners and hospital doctors, particularly the Northern Region Haematologists Group, for taking the time and trouble to provide us with information. We are also grateful to the regional immunology laboratory at the Churchill Hospital, Oxford, for their assistance with the antibody assays, and particularly to Dr Helen Griffiths for technical advice. We thank David Brownlee of Bayer plc for arranging the printing of the patient warning cards.

\section{Appendix 1}

Suggested guidelines for dealing with asplenia/ hyposplenia, Newcastle General Hospital Patients with asplenia or hyposplenia (sickle cell disease, some patients with coeliac disease) are at increased risk of overwhelming bacterial infection. The onset of symptoms may be extremely rapid and death may occur in hours. The risk does not diminish with time after splenectomy. It is highest in those who have had a splenectomy for lymphoproliferative disease (nonHodgkin lymphoma, chronic lymphatic leukaemia) or who have concomitant liver disease.

1. All patients regardless of underlying condition should be on lifelong antibiotic prophylaxis.

2 . In the under fives, children and adults this should be either penicillin $\mathrm{V}$ twice daily or amoxycillin once daily, with a preference for penicillin V. Cotrimoxazole may be an alternative for children, especially those with congenital asplenia, but it is not recommended for adults.

Adult doses: penicillin V $250 \mathrm{mg}$ twice daily; amoxycillin $500 \mathrm{mg}$ once daily. Children's dosage should be discussed with paediatric immunologists,

3. For penicillin allergic patients erythromycin should be used (250 mg twice daily, adult dose).

4. Patients undergoing elective splenectomy should receive Pneumovax, Hib, and meningococcal polysaccharide vaccines at least one week before surgery.

5. All other patients should receive the three vaccines even after splenectomy as there may be some benefit.

6. Blood levels of antibodies to these polysaccharides should be measured three weeks after surgery and then at yearly intervals.

7. Booster immunisation may be required if levels of antibodies fall below protective levels.

8. All adults should receive an annual influenza immunisation.

9. Patients travelling to malarial areas and areas where penicillin resistant pneumococci have been found require specialist advice.

10. Any asplenic/hyposplenic patient who develops a sudden febrile illness should be treated promptly with full dose antibiotics. The onset of illness may be extremely rapid and the speed of response may determine outcome

Intravenous penicillin after blood culture (if possible) should be started at once if the clinical circumstances warrant it and the patient referred to the nearest acute hospital.

11. Annual follow up is recommended, to reinforce the need for prophylaxis and to check antibody and immunisation status.

\section{Appendix 2}

The personalised laminated warning card used in the register (both sides shown).

The carrier of this Card suffers from ASPLENIA or has undergone a SPLENECTOMY. There is an increased risk of infection.

Please ensure that you carry it with you at all times. If you are ill or need to see your doctor for any other reason, please show him this card.

Name:

Address:

General Practitioner:

Surgery Telephone No:

Hospital Consultant:

Hospital:

Reason for Splenectomy:

PTO

As you do not have a spleen, it is important that you remember to:

1. Take your antibiotic tablets regularly, as instructed by your doctor.

2. Ensure that you get regular immunisations, as recommended by your doctor.

3. Discuss any foreign travel in advance with your doctor: Special precautions may be required and your doctor may advise a change of antibiotic tablets.

If you become suddenly unwell with a high temperature, shivering or shaking, and feel dizzy or faint, you should:

1. Immediately take a double dose of your normal antibiotic.

2. Contact your own doctor at once.

3. If your own doctor is not available or there is any delay, go at once to the nearest hospital. Show the hospital doctors this card and your normal antibiotic tablets. 
1 McMullin M, Johnston G. Long term management of patients after splenectomy. BMF 1993;307:1372-3.

portan JE. Management of asplenic patients. Br f Haematol 1993;84:566-9.

3 Mayon-White R. Protection for the asplenic patient. Prescribers F 1994;34:165-70.

4 Read RC, Finch RG. Prophylaxis after splenectomy. $f$ Antimicrob Chemother 1994;33:4-6.

5 Cullingford GL, Watkins DN, Watts ADJ, et al. Severe late postsplenectomy infection. Brf S Surg 1991;78:716-21.

6 Pimpl W, Dapunt O, Kaindl H, et al. Incidence of septic and thromboembolic-related deaths after splenectomy in adults. Br 7 Surg 1989;76:517-21.

7 Holdsworth RJ, Irving AD, Cuschieri A. Postsplenectomy sepsis and its mortality rate: actual versus perceived risks. Br F Surg 1991;78:1031-8.

8 Evans D. Postsplenectomy sepsis 10 or more years after operation. f Clin Pathol 1985;38:309-11.

9 Reid MM. Splenectomy, sepsis, immunisation and guidelines. Lancet 1994;344:970-1.

10 Working Party. Guidelines for the prevention and treatment of infection in patients with an absent or dysfunctional spleen. Working Party of the British ards in Haematology Clinical Haematology Task Force. BMF 1996;312:430

11 Hassan IS, Snow MH, Ong EL. Overwhelming pneumococcal sepsis in two patients splenectomised more than ten years previously. Scot Med f 1996;41:17-19

12 Hargreaves RM, Lea JR, Griffiths $\mathrm{H}$, et al. Immunological factors and risk of infection in plateau phase myeloma. $\mathcal{F}$ Clin Pathol 1995; 48:260-6.
13 Booy R, Taylor SA, Dobson SRM, et al. Immunogenicity and safety of PRP-T conjugate vaccine given according to the British accelerated Child 1992;67:475-8.

14 MacInnes J, Waghorn DJ, Haworth E. Management of asplenic patients in South Buckinghamshire: an audit of local practice. CDR Rev 1995;5:R173-7.

15 Konradsen HB, Rasmussen C, Ejstrud P, et al. Antibody evels against Streptococcus pneumoniae and Haemophilus influenzae type $b$ in a population of splenectomised individuals with varying vaccination status. Epidemiol Infect 1997; 119:167-74.

16 Gaines $\mathrm{AD}$, Buckley $\mathrm{RH}$. Impaired antibody response to polysaccharides in association with functional asplenia. $\mathcal{F}$ Pediatr 1989;114:89-91.

17 McElroy PJ, Henderson FI, Brown DL. Immune status and response to immunisation with polysaccharide vaccines of a healthy congenitally asplenic woman. Clin Exp Immunol 1989;78:402-5.

18 Sullivan JL, Ochs HD, Schiffman G, et al. Immune response after splenectomy. Lancet 1978;i:178-81.

19 Siber GR, Gorham C, Marten P, et al. Antibody response to pre-treatment immunisation and post-treatment boosting with bacterial polysaccharide vaccines in patients with Hodgkin's disease. Ann Intern Med 1980;104:467-75.

20 Mufson MA, Krause HE, Schiffman G, et al. Pneumococcal antibody levels one decade after immunisation of healthy adults. Am f Med Sci 1987;293:279-84.

21 Konradsen HB, Pederson FK, Henrichsen J. Pneumococcal vaccination of splenectomised children. Pediatr Infect Dis $\mathcal{F}$ 1990;9:258-63. 